\title{
Correction to: Identification of appropriate reference genes for human mesenchymal stem cell analysis by quantitative real-time PCR
}

\author{
Xiuying Li • Qiwei Yang · Jinping Bai - Yali Xuan · Yimin Wang
}

Published online: 8 October 2021

(C) Springer Nature B.V. 2021

Correction to: Biotechnology Letters (2015) 37:67-73

https://doi.org/10.1007/s10529-014-1652-9

In the original publication of the article, some of the graphs in Fig. 1 were published incorrectly. The correct graphs are given below:
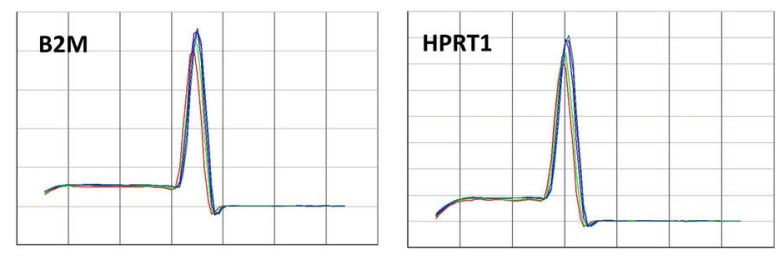

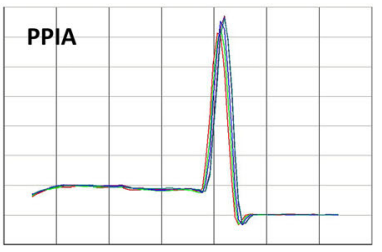

Publisher's Note Springer Nature remains neutral with regard to jurisdictional claims in published maps and institutional affiliations.
The original article can be found online at https:// doi.org/10.1007/s10529-014-1652-9.

Supplementary Information The online version contains supplementary material available at https://doi.org/10.1007/ s10529-021-03187-x.

X. Li · Q. Yang $\cdot$ Y. Wang $(\bowtie)$

The Central Laboratory, China-Japan Union Hospital, Jilin University, 126 Xiantai Street, Changchun 130033, Jilin, China

e-mail: yiminwang@hotmail.com

\section{J. Bai}

Department of Pathology, The Key Laboratory of Pathobiology, Jilin University, Ministry of Education, 126 Xinmin Street, Changchun 130021, Jilin, China
Y. Xuan

Princess Margaret Hospital, 610 University Ave, Toronto, ON M5G 2M9, Canada

Y. Wang

Jilin Zhongke Bio-Engineering Co., Ltd, Building 2, 3355

Guigu Street, Changchun 130012, Jilin, China 